\title{
Transcriptome profiling of the fertile parent and sterile hybrid in tea plant flower buds
}

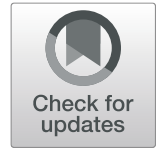

\author{
Linbo Chen ${ }^{1,2+}$, Hao Qu ${ }^{1,2+}$, Lifei Xia ${ }^{1,2}$, Yue Liu ${ }^{1,2}$, Huibing Jiang ${ }^{1,2}$, Yunnan Sun ${ }^{1,2}$, Mingzhi Liang ${ }^{1,2^{*}}$ (i) and \\ Changjun Jiang ${ }^{3 *}$
}

\begin{abstract}
Background: The tea plant is a crucial economic crop. The floral organ development consumes a large amount of nutrients, which affects the leaf yield. To understand the mechanism by which the tea plant produces sterile floral buds, we obtained a sterile tea plant by artificial hybridization. RNA-sequencing based transcriptome analysis was implemented in three samples to determine the differentially expressed genes (DEGs) related to flower development.

Results: In this study, a total of 1991 DEGs were identified; 1057 genes were up-regulated and 934 genes were downregulated in sterile hybrid floral buds. These were mainly distributed in the regulation of biological and metabolic processes. Significantly, auxin biosynthesis genes YUCCA, AUX1 and PIN were dramatically down-regulated, and ARF gene was up-regulated in the sterile hybrid floral buds, and flower development-related genes AP1, AP2 and SPL were changed. A total of 12 energy transfer-related genes were significantly decreased. Furthermore, the expression of 11 transcription factor genes was significantly different.

Conclusion: The transcriptome analysis suggested that the production of sterile floral buds is a complex bioprocess, and that low auxin-related gene levels result in the formation of sterile floral buds in the tea plant.
\end{abstract}

Keywords: Tea plant, Sterile floral buds, Differentially expressed genes, Auxin

\section{Introduction}

Sterility is a complex phenomenon in plants, the main factors of which include the accumulation of reactive oxygen species, abnormalities in energy metabolism, programmed cell death, imbalance in endogenous hormones and changes in environmental condition [1-3]. In Arabidopsis and cereal grains, floral organ degeneration and abiotic stresses result in sterility. Abortion or degeneration of developing stamens and pistil is the key mechanism used by plants to produce sterile flowers [4].

Among the sterility mechanisms of the plant, flower development is one of the main concerns. It requires early and later organ identity genes. PINFORMED (PIN1), PINOID (PID), YUCCA (YUC) and NAKED PINS IN YUC MUTANT (NPY) are necessary for flower development

\footnotetext{
*Correspondence: ynnkycys@126.com; jiangcj@ahau.edu.cn

† Linbo Chen and Hao Qu contributed equally to this work.

${ }^{1}$ Tea Research Institute, Yunnan Academy of Agricultural Sciences, Menghai 666201, China

${ }^{3}$ State Key Laboratory of Tea Plant Biology and Utilization, Anhui Agricultural University, Hefei 230036, China

Full list of author information is available at the end of the article
}

[5, 6]. Organ identity genes APETALA1 (AP1), APETALA2 (AP2), APETALA3 (AP3), PISTILLATA (PI) and AGAMOUS ( $A G$ ) are essential for the ABC model in Arabidopsis [7]. The mutation of these genes causes more petals, fewer stamens, fused floral organs, and valveless gynoecia. In addition, important genes for flower development include SPL, TPD1, AMS, DYT1, SHP, WUS [8-13]. Brassionsteroid and jasmonic acid play a positive role in promoting the formation of stamens and pollen. Gibberellin deficiencies are related to male sterility, and the formation of female flowers requires the presence of ethylene [14-16]. Moreover, flower development is regulated by the coordinated interaction of the transcription factor LEAFY and auxin [17]. However, molecular data are limited for the tea plant; the molecular mechanism of flower development remains unknown.

The tea plant is a crucial cash crop widely distributed around the world. Tea leaves have been used to produce various tea beverages. The floral organ development results in the fall of the yield of tea leaves by consuming a large amount of nutrients. Cultivation of the sterile tea plant is key to increasing the yield. The tea plant is self-incompatible, 
Table 1 Primer Sequences for q-PCR

\begin{tabular}{ll}
\hline Gene name & Primer Sequence (5' to $3^{\prime}$ ) \\
\hline AP2 & F:TACAGAGGAGTAACAAGGCATCA \\
& R:CGTCAAAGTCGTCACAGCA \\
& F:GCTTCCACAACTCAACTCCAGA \\
& R:CAACAAGGCTCGTGAAATCG \\
ARF & F:TGAAACAGAGGAGTCAGGCAA \\
& R:CCAGTCTCATCCCACTCTACCT \\
IAA7 & F:TCCAATGAGAAGAAAGACCCTG \\
& R:CACCTTCACAAACGCCACA \\
AUX1 & F:ACTGAGGCTGAGGTTGGTGA \\
& R:TTAGATTGATGGGCGTGGT \\
ATL3 & F:CACACTAACCCTACCATCAGCA \\
& R:CAGTGTCTCTGAAACCAGTCCT \\
GAPDH & F:GATAGTGTCACGGTCAATGGA \\
& R:GCAGCAGCCTTATCCTTATCAG \\
\hline
\end{tabular}

thus we performed transcriptome sequencing and comparative analysis on three samples, including Foxiang2 (FBH), Fudingbaicha $(\mathrm{MBH})$ and hybrid sterile flowers (ZDH). The aim was to analyze the differentially expressed genes between the fertile and sterile floral buds, and to identify their related bioprocesses and correlative factors. Our results will help to reveal important information on the mechanism of sterility in the tea plant.

\section{Materials and methods}

\section{Plant materials}

The plant materials were five-year-old tea plants $(C$. sinensis (L.) O. Kuntze) from the Tea Research Institute, Yunnan Academy of Agricultural Sciences called 'Foxiang2', used as FBH, 'Fudingbaicha', used as MBH, and a sterile hybrid, used as ZDH. Flower buds were separately stripped from three tea plants. All of the experiments were performed using three biological replicates. Flower buds were picked on October 16, 2016, after which they were frozen using liquid nitrogen and stored in a freezer at $80^{\circ} \mathrm{C}$ for subsequent mRNA analysis.

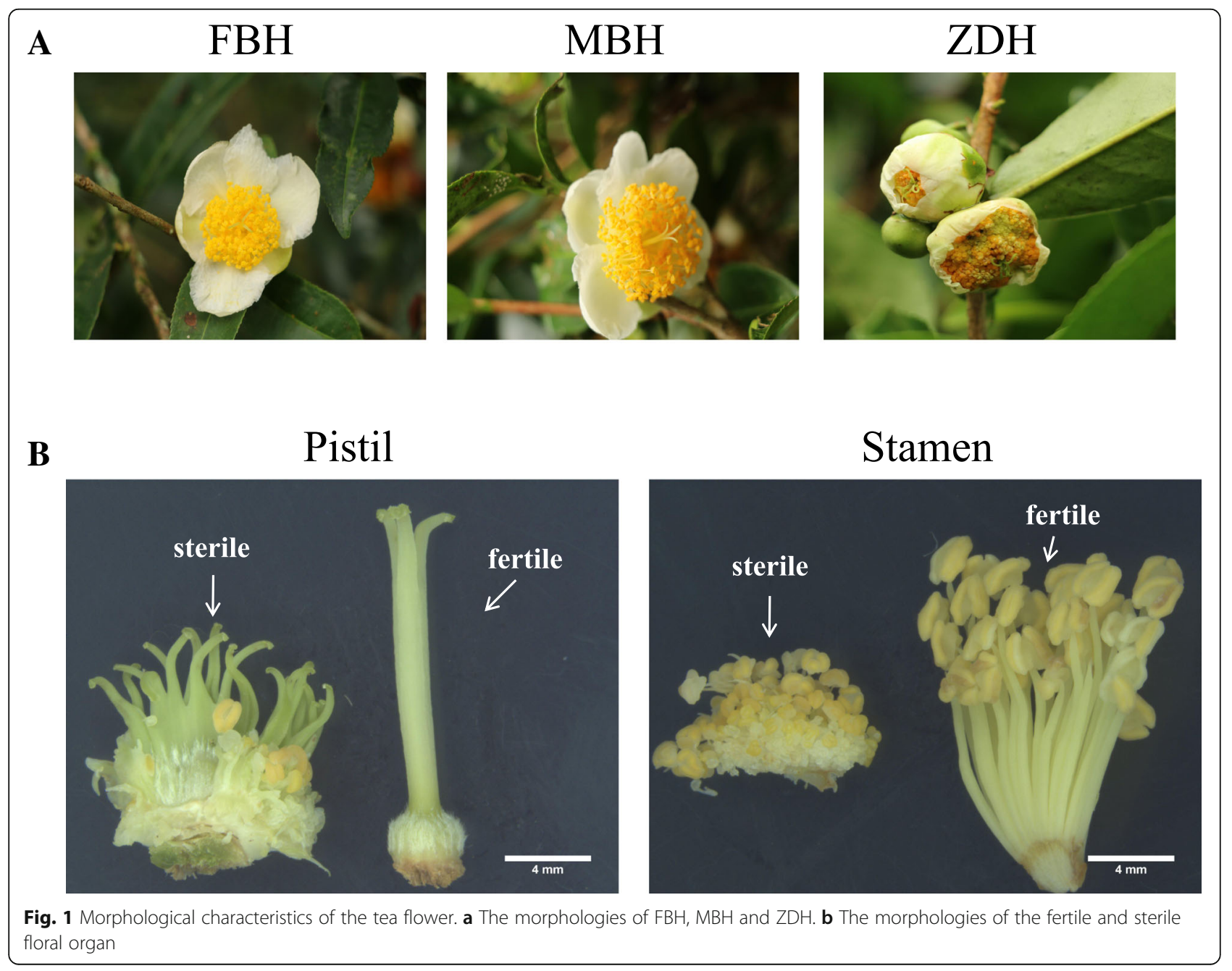


Table 2 Summary Dataset of Transcriptome Assembly

\begin{tabular}{llll}
\hline & male parent $(\mathrm{FBH})$ & female parent $(\mathrm{MBH})$ & sterile flowers $(\mathrm{ZDH})$ \\
\hline Clean reads & $64,267,724$ & $60,674,496$ & $74,575,474$ \\
GC content & $44.00 \%$ & $44.17 \%$ & $43.67 \%$ \\
Q30 & $92.41 \%$ & $92.09 \%$ & $92.53 \%$ \\
Mapped reads ratio & $73.64 \%$ & $75.27 \%$ & $71.43 \%$ \\
\hline
\end{tabular}

\section{CDNA library construction and sequencing}

The construction of cDNA libraries and transcriptome sequencing were completed by Beijing Novogene Technology (Beijing, China). Total RNA was used as the starting sample and was directly added to the 3 '-terminal hydroxyl group and the uniquely structured complete phosphoryl groups at the $5^{\prime}$-terminal of the sRNA, followed by reverse transcription to synthesize cDNA. After polymerase chain reaction amplification, polyacrylamide gel electrophoresis was used to separate the target DNA fragments, and the gel was recovered, completing the cDNA library. The effective concentration of the library was $>2 \mathrm{nmol} / \mathrm{L}$, and sequencing was performed using Illumina HiSeq 2000 after the library was certified. All of the experiments were performed using three replicates.

\section{Transcriptome assembly}

The original image data files obtained from the Illumina HiSeq 2000 were subjected to base calling analysis and converted into raw reads. Among the raw reads obtained from the sequencing, the low-quality reads with adaptors were processed to obtain clean reads. The clean reads were assembled separately, and TGICL was used to get the longest non-redundant unigenes. The transcriptome data were deposited to the NCBI SRA database (SRA accession: PRJNA503652).

\section{Differentially expressed genes (DEGs) test}

Differentially expressed genes (DEGs) analysis using DEGseq (three biological replicates per group). DESeq provides statistical routines for determining differential
A

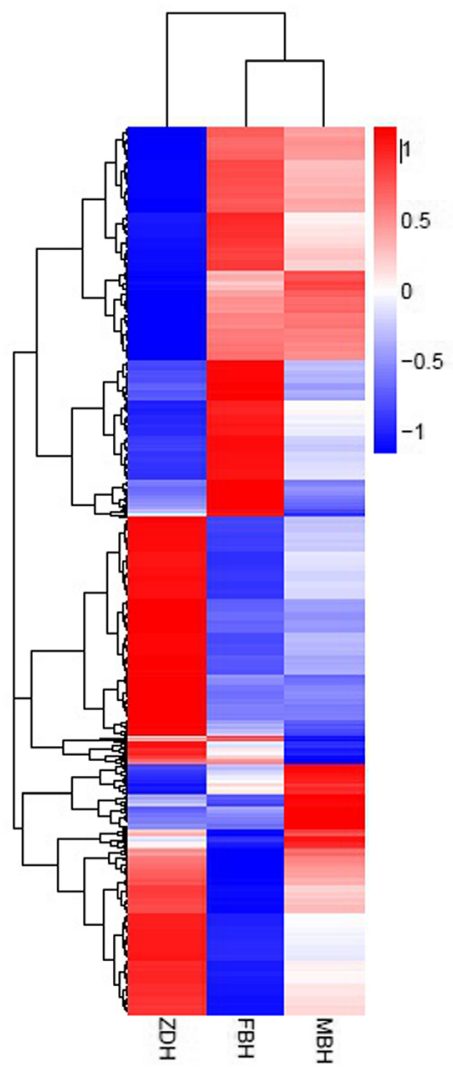

B

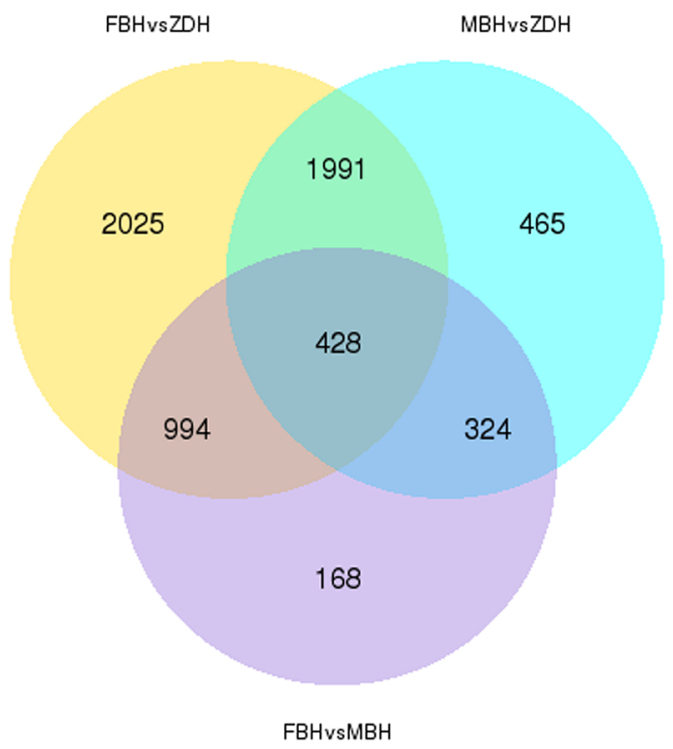

Fig. 2 Cluster analysis of DEGs. a Heat map showing expression of the DEGs. High expression genes appear red, while low expression genes appear blue on the heat map. $\mathbf{b}$ The Venn diagram showing the number of DEGs between FBHvsZDH, MBHvsZDH, FBHvsMBH 
Gene Function Classification (GO)

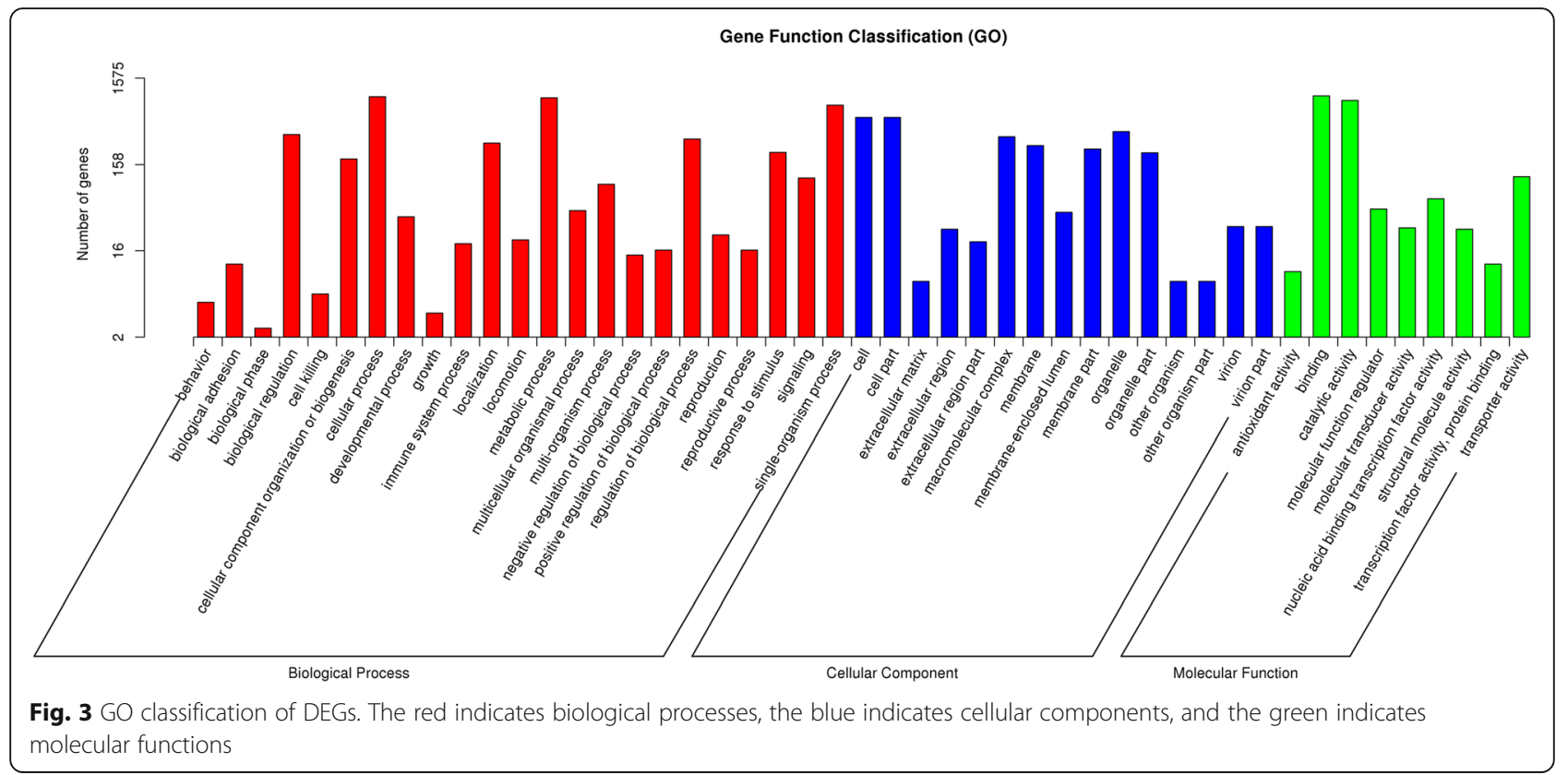

expression in digital gene expression data, using a model based on negative binomial distribution. The resulting $P$-values were adjusted using the Benjamin and Hochberg approach for controlling the false discovery rate. The genes with P-value $<0.05$ found by DESeq were differentially expressed.

\section{GO and KEGG analysis}

DEGs were characterized according to Gene Ontology enrichment analysis. GO annotations were provided by the Blast2GO program. Then the GO classification graph was generated by the WEGO.

KEGG was used to analyze the biological process and unigenes annotation of pathway. The results were comparatively analyzed between the KEGG integrated database resource and our data.

\section{Quantitative real-time PCR assays}

Total RNA was isolated using TRlpure reagent (BioTeke, China) according to the manufacturer's instructions. cDNA was synthesized from total RNA using a PrimeScript RT reagent kit (TaKaRa, Japan). The obtained cDNA was used as a template in SYBR green-based q-PCR (CFX-96, Bio-Rad, Hercules, CA, USA). GAPDH was used for normalization. The primers are shown in Table 1.

\section{Results}

\section{Morphological characteristics of sterile flower buds}

The floral organ of the tea plant consists of a complete bisexual flower composed of a thalamus, calyxes, petals, stamens, and a pistil (Fig. 1a). In our study, the sterile flower buds were smaller than the male and female parent flower. Petals were improperly unfolding during the developmental process until flower buds abscission (Fig. 1a). The filaments were shorter than those of fertile flowers, and the anthers contained no pollen (Fig. 1b). In addition, sterile flowers contained two to four imperfect pistils, which were split into two to five smaller stigma (Fig. 1b).

\section{Transcriptome profiling of the male/female parent and the hybrid bud}

We used the Illumina HiSeq 2000 platform to create three cDNA libraries, $\mathrm{FBH}, \mathrm{MBH}$, and $\mathrm{ZDH}$. As a result, a total of $64.2,60.6$ and 74.6 million clean reads were generated. The quality score (Q30) percentage was above $92 \%$, with the GC content of each clean data above $43 \%$. The ratio of mapped reads was 73.64, 75.27 and $71.43 \%$, respectively (Table 2). Afterwards, Trinity was used in splicing for clean reads. A total of 268,289 transcripts were obtained, and the longest transcript for each gene was selected as the unigene, of which 173,248 were screened for differential expression analysis (DEGs).

\section{DEGs and functional characterization}

A total of 6395 DEGs were detected in $\mathrm{FBH}, \mathrm{MBH}$ and ZDH. 1914 DEGs were screened out between FBH and $\mathrm{MBH}$. Between $\mathrm{ZDH}$ and $\mathrm{FBH}$, the number of DEGs was 5438, whereas it was 3208 between $\mathrm{ZDH}$ and $\mathrm{MBH}$. 1991 DEGs were screened out in $\mathrm{ZDH}$, the expression quantity was different with $\mathrm{FBH}$ and $\mathrm{MBH}$ (Fig. 2).

The unique DEGs were characterized using the GO and KEGG databases. The GO enrichment analysis categorized 1991 unigenes, which contained 47 subcategories of biological processes, cellular components, and molecular functions. For the biological process, "biological regulation", "cellular process", "metabolic process", and "single 


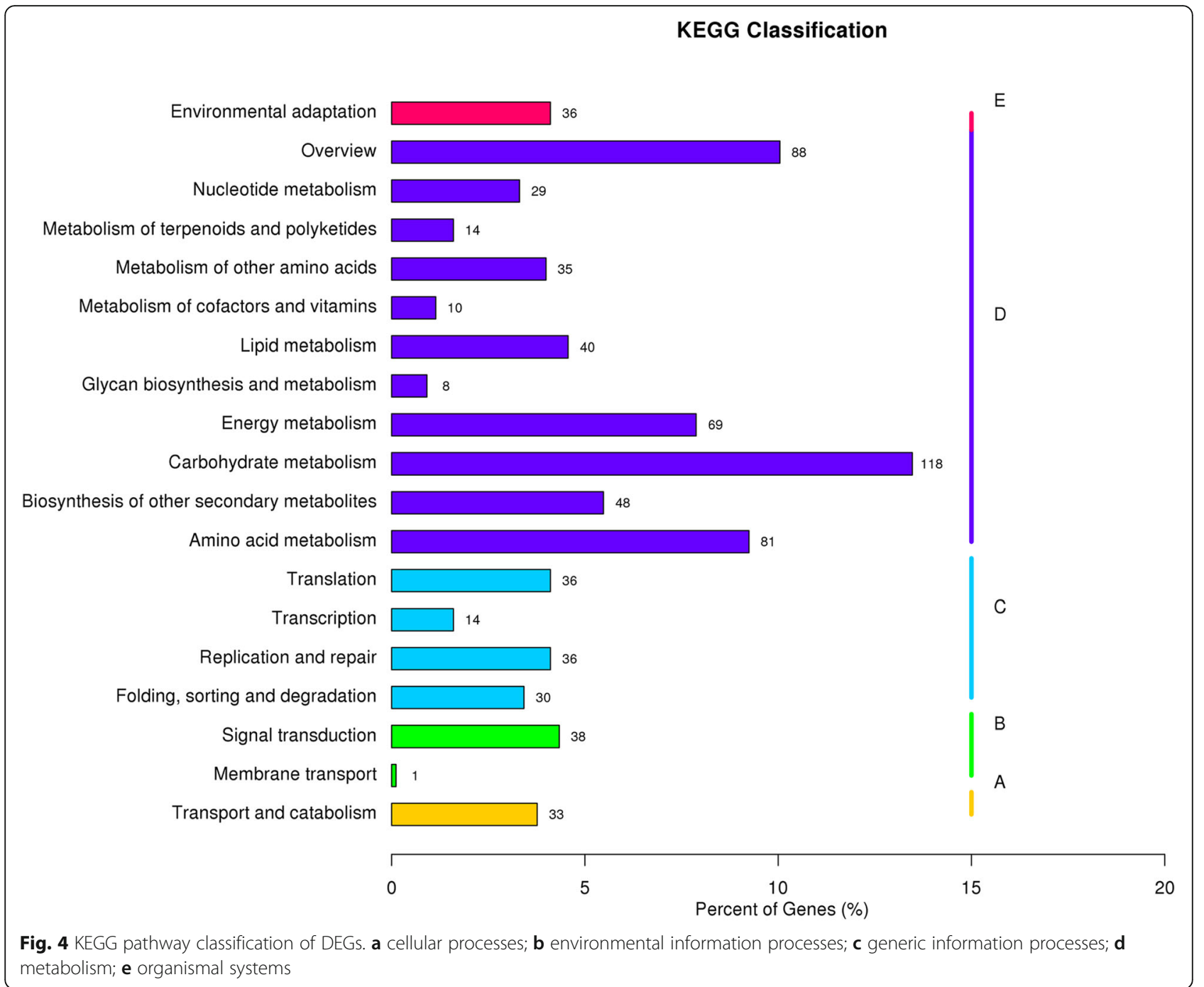

organism process" were the most representative groups. In the subcategory of cellular component, "cell", "cell part", "macromolecular complex", and "organelle" were the predominant groups. For the molecular function, "binding", and "catalytic activity" were the most common. Moreover, "reproduction", "reproductive process", and "growth" were enriched in 24, 16, and 3 unigenes, respectively (Fig. 3).

The KEGG analysis revealed that 764 unigenes were mapped into KEGG, containing cellular processes, environmental information processes, generic information processes, metabolism, and organismal systems. The main KEGG pathways included "Carbon metabolism", "Biosynthesis of amino acids", "Starch and sucrose metabolism", "Plant hormone signal transduction", "Plant pathogen interaction", and "Glycolysis/Gluconeogenesis" (Fig. 4).

\section{Identification of auxin-related genes involved in DEGs}

Auxin is essential for flower organ development [18]. Among DEGs, the auxin biosynthesis gene YUCCA related to floral organ formation was down-regulated. The auxin flux-related PIN homolog gene related to gynoecium formation was down-regulated. In addition, we identified ten auxin response factors ARF (Cluster-23,036.113917, Cluster-23,036.14480, Cluster-23,036.14481, Cluster-23,036.85241, Cluster-23,036.27862, Cluster-23,036.29441, Cluster-23,036.70956, Cluster-23,036.66525, Cluster-23,036.54073 and Cluster-23,036.87364) related to flower maturation was up-regulated (Table 3).

\section{Identification of flower development-related genes involved in DEGs}

Abnormal flower development is the key factor responsible for the sterility of the tea plant. The formation of flowers is a key step in the plant life cycle, which is a complex process [19]. Each stage of the process is regulated by flower development-related genes, specifically the $\mathrm{ABC}$ floral organ-identity genes [20]. We identified the A-class genes $A P 1$ and $A P 2$ were up-regulated.In 
Table 3 DEGs Related to IAA Signal Transduction

\begin{tabular}{|c|c|c|c|c|}
\hline \multirow[t]{2}{*}{ Gene ID } & \multirow[t]{2}{*}{ KO name } & \multirow[t]{2}{*}{ Annotation } & \multicolumn{2}{|l|}{$\log _{2}$ ratio } \\
\hline & & & FBH vs ZDH & $\mathrm{MBH}$ vs $\mathrm{ZDH}$ \\
\hline Cluster-23,036.78079 & YUCCA & indole-3-pyruvate monooxygenase YUCCA10 & 2.50 & 2.57 \\
\hline Cluster-23,036.68881 & JAR1 & Indole-3-acetic acid-amido synthetase GH3.5 & 4.81 & 4.59 \\
\hline Cluster-23,036.78790 & ILR1 & IAA-amino acid hydrolase ILR1-like 6 & 2.65 & 2.00 \\
\hline Cluster-23,036.63096 & ILR1 & IAA-amino acid hydrolase ILR1-like 4 & 4.29 & 3.59 \\
\hline Cluster-23,036.77706 & PIN & auxin efflux carrier protein & 3.34 & 2.87 \\
\hline Cluster-23,036.72193 & AUX1/LAX & auxin transporter-like protein 4 & 2.45 & 2.44 \\
\hline Cluster-23,036.74178 & AUX1/LAX & auxin transporter-like protein 3 & 2.99 & 2.69 \\
\hline Cluster-23,036.53622 & Aux/IAA & Auxin-responsive protein IAA7 & 6.17 & 5.43 \\
\hline Cluster-23,036.68884 & Aux/IAA & auxin-induced protein 22D-like & 3.48 & 3.33 \\
\hline Cluster-23,036.74950 & Aux/IAA & auxin-induced protein 22D-like & 5.43 & 5.34 \\
\hline Cluster-23,036.113917 & ARF & auxin response factor 9 & -4.76 & -4.09 \\
\hline Cluster-23,036.14480 & ARF & auxin response factor 5 & -7.27 & -4.02 \\
\hline Cluster-23,036.14481 & ARF & auxin response factor 5 & -6.00 & -4.96 \\
\hline Cluster-23,036.85241 & ARF & auxin response factor 4 & -2.60 & -2.02 \\
\hline Cluster-23,036.27862 & ARF & auxin response factor 28 & -3.23 & -2.24 \\
\hline Cluster-23,036.29441 & ARF & auxin response factor 28 & -1.90 & -1.09 \\
\hline Cluster-23,036.70956 & ARF & auxin response factor 2 & -1.41 & -1.11 \\
\hline Cluster-23,036.66525 & ARF & auxin response factor 19-like & -1.38 & -1.26 \\
\hline Cluster-23,036.54073 & ARF & auxin response factor 19-like & -1.68 & -1.49 \\
\hline Cluster-23,036.87364 & ARF & auxin response factor 1 isoform $X_{1}$ & -1.98 & -1.77 \\
\hline
\end{tabular}

addition, flower development-related genes, such as floral organ formation SPL homolog genes (Cluster-23,036.89600, Cluster-23,036.17164, Cluster-23,036.96383, Cluster23,036.48034 and Cluster-23,036.89141) were up-regulated (Table 4).

\section{Identification of energy transfer-related genes in DEGs} Energy transfer is an important process of plant growth and development. It is the foundation of the plant's life
[21]. We identified that some genes involved in energy transfer were differentially expressed, including $A B C$ transporter $\mathrm{B}$ family member $1(A B C B 1)$, six transporting ATPase-related genes (ATPeFOD, ATPeFOO, ATPeF1B, $A T P e V 0 A, A T P e V 1 C$, and $A T P e V 1 B)$, and five solute carrier-related genes (SLC2A8, SLC15A3, SLC25A11, $S L C 32 A$, and $S L C 35 B 3$ ) (Table 5). A total of 12 genes were down-regulated. In particular, transporting ATPase subunit beta $(A T P e F 1 B)$, solute carrier family $32(S L C 32 A)$ and

Table 4 DEGs Related to Flower Development

\begin{tabular}{lllll}
\hline Gene ID & KO & Annotation & Log $_{2}$ ratio \\
\cline { 3 - 5 } & name & & FBH vs ZDH & MBH vs ZDH \\
\hline Cluster-23,036.89600 & SPL8 & squamosa promoter-binding-like protein 8 & -3.52 & -2.36 \\
Cluster-23,036.17164 & SPL6 & squamosa promoter-binding-like protein 6 & -2.32 & -2.00 \\
Cluster-23,036.96383 & SPL3 & squamosa promoter-binding-like protein 3 & -4.85 & -3.30 \\
Cluster-23,036.48034 & SPL12 & squamosa promoter-binding-like protein 12 & -1.68 & -2.86 \\
Cluster-23,036.89141 & SPL9 & squamosa promoter-binding-like protein 9 & -2.75 & -3.19 \\
Cluster-23,036.69978 & AP1 & MADS-box transcription factor APETALA1-like & -1.92 & -1.36 \\
Cluster-23,036.57048 & AP2 & AP2-like ethylene-responsive transcription factor RAP2-7 & -2.32 & -2.26 \\
Cluster-23,036.116884 & AP2 & AP2-like ethylene-responsive transcription factor ANT & -8.76 & -7.94 \\
Cluster-23,036.90254 & AGO5 & protein argonaute 5 & -3.32 & -4.08 \\
Cluster-23,036.85203 & AGO4 & protein argonaute 4 & -2.48 & -1.69 \\
Cluster-23,036.99883 & AGO2 & protein argonaute 2 & -1.61 & -7.52 \\
Cluster-23,036.95433 & AGO10 & protein argonaute 10 & -3.76 & -2.27 \\
\hline
\end{tabular}


Table 5 DEGs Related to Energy Transfer

\begin{tabular}{lllll}
\hline Gene ID & KO name & Annotation & Log $_{2}$ ratio \\
\cline { 3 - 5 } & & & FBH vs ZDH & MBH vs ZDH \\
\hline Cluster-23,036.67567 & ABCB1 & ABC transporter B family member 1 & 1.86 & 1.56 \\
Cluster-23,036.73251 & ATPeF0D & transporting ATPase subunit d & 1.82 & 1.85 \\
Cluster-23,036.68425 & ATPeF0O & transporting ATPase subunit O & 6.03 & 1.71 \\
Cluster-23,036.67744 & ATPeF1B & transporting ATPase subunit beta & 1.92 & 6.58 \\
Cluster-23,036.71789 & ATPeV0A & transporting ATPase subunit a & 1.77 & 1.46 \\
Cluster-23,036.61724 & ATPeV1C & transporting ATPase subunit C & 1.49 & 1.37 \\
Cluster-23,036.73958 & ATPeV1B & transporting ATPase subunit B & 2.86 & 1.21 \\
Cluster-23,036.75094 & SLC2A8 & solute carrier family 2 & 1.87 & 2.31 \\
Cluster-23,036.55114 & SLC15A3 & solute carrier family 15 & 2.48 & 1.49 \\
Cluster-23,036.73478 & SLC25A11 & solute carrier family 25 & 5.92 & 1.87 \\
Cluster-23,036.72951 & SLC32A & solute carrier family 32 & 5.77 & 5.15 \\
Cluster-23,036.49241 & SLC35B3 & solute carrier family 35 & 6.03 \\
\hline
\end{tabular}

solute carrier family 35 (SLC35B3) were down-regulated 65.7, 60.9 and 54.9-fold, respectively, in ZDH compared with FBH. The expression levels of ATPeF1B, SLC32A and SLC35B3 were also down-regulated 96.8, 34.7 and 65.4-fold, respectively, in ZDH compared with $\mathrm{MBH}$.

\section{Identification of other transcription factor genes in DEGs}

We also identified some transcription factor genes. Among these genes, the expression levels of transcription factor AS1 (AS1), transcription factor CPC $(C P C)$, and nuclear transcription factor $\mathrm{Y}(N F Y A)$ were up-regulated significantly. Furthermore, MADS-box transcription factor (K09264), two transcription factor bHLH (bHLH77 and bHLH79), three transcription factor MYB (GAMYB, MYBP and MYB21), transcription factor TGA6 (TGA6) and transcription factor GTE2 (GTE2) were down-regulated in ZDH (Table 6).

\section{qPCR analysis of DEGs}

To confirm the transcriptome sequencing results, six DEGs were selected for $\mathrm{qPCR}$ analysis. Among these genes, Cluster-23,036.57048 (AP2) and Cluster-23,036.14480 $(A R F)$ genes were up-regulated, and Cluster-23,036.68881 (JAR1), Cluster-23,036.53622 (IAA7), Cluster-23,036.69380 (AUX1) and Cluster-23,036.74178 (ATL3) genes were down-regulated (Fig. 5). qRT-PCR results showed a consistent expression tendency compared with the RNA-Seq. The result further confirmed the reliability and accuracy of the transcriptome sequencing.

\section{Discussion}

Here, we used transcriptome sequencing to explore candidate genes associated with sterile floral buds in the tea plant. The method has been applied to the study of the genome for the tea plant [22-25]. A total of 1991 DEGs

Table 6 DEGs Related to Transcription factors

\begin{tabular}{lllll}
\hline Gene ID & KO & Annotation & Log $_{2}$ ratio \\
\cline { 3 - 5 } & name & & -2.43 & MBH vD \\
\hline Cluster-23,036.105297 & AS1 & Transcription factor AS1 & -1.6 \\
Cluster-23,036.65747 & K09264 & MADS-box transcription factor, plant & 1.63 & 1.48 \\
Cluster-23,036.17098 & CPC & Transcription factor CPC & -3.25 & -3.1 \\
Cluster-23,036.14144 & NFYA & nuclear transcription factor Y, alpha & -5.52 & -6.14 \\
Cluster-23,036.62850 & bHLH77 & Transcription factor bHLH77 & 2.68 & 2.92 \\
Cluster-23,036.69683 & bHLH79 & Transcription factor bHLH79 & 6.53 & 5.59 \\
Cluster-23,036.81661 & GAMYB & Transcription factor GAMYB & 2.46 & 2.42 \\
Cluster-23,036.56315 & GTE2 & Transcription factor GTE2 & 5.82 & 7.47 \\
Cluster-23,036.76553 & MYBP & Transcription factor MYB108 & 6.92 \\
Cluster-23,036.71827 & MYB21 & Transcription factor MYB21 & 4.64 & 5.01 \\
Cluster-23,036.70389 & TGA6 & Transcription factor TGA6 & 5.48 & 5.09 \\
\hline
\end{tabular}




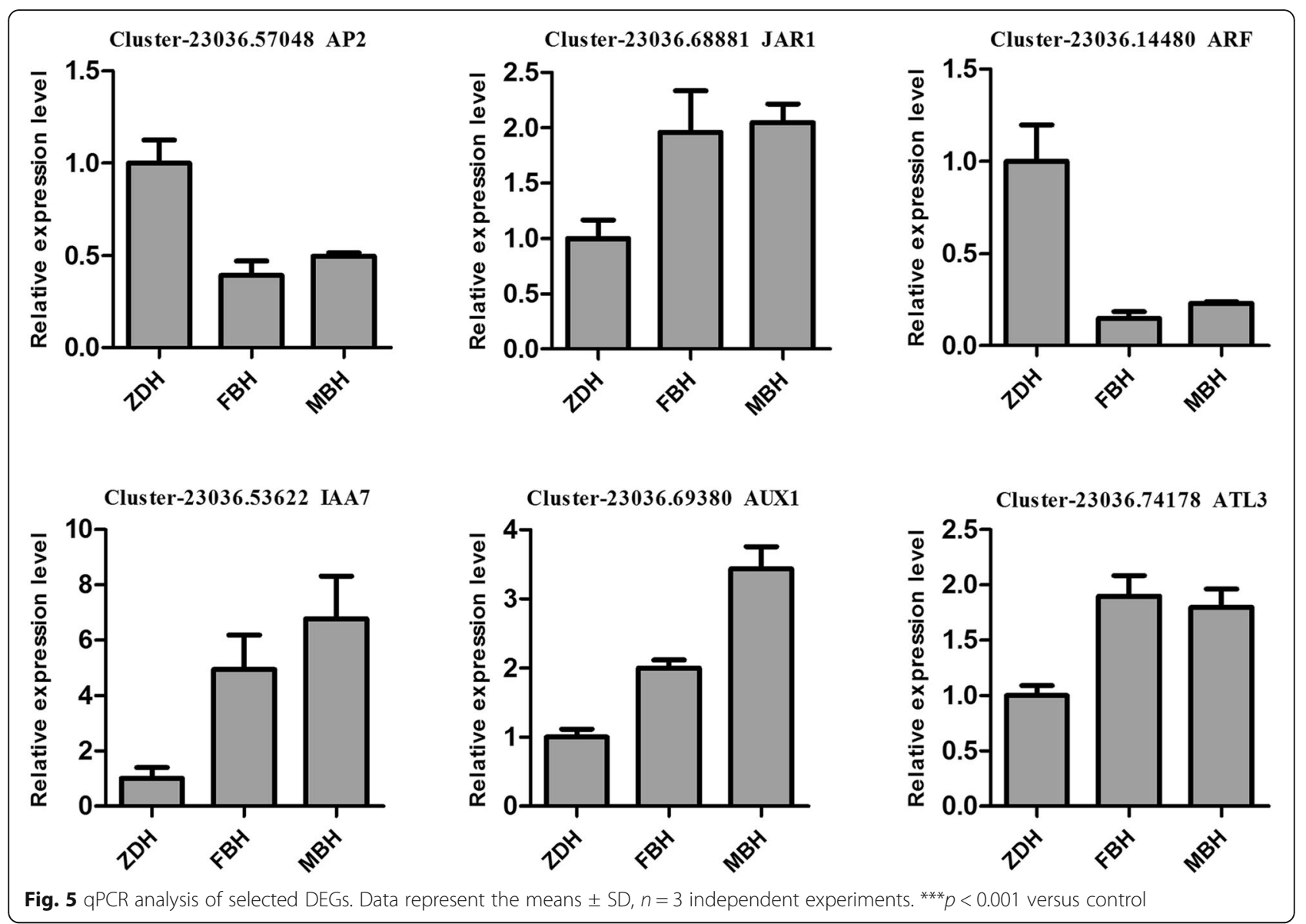

were screened out from the comparison among the three cDNA libraries (Fig. 2). The complexity of DEGs function was demonstrated by Go and KEGG analysis (Figs. 3 and 4). Based on the analysis, 452 metabolism-related pathways were identified (Fig. 4), suggesting that metabolism may be crucial for the sterility mechanism in the tea plant.

In general, the flavin monooxygenase (YUCCA) is involved in the tryptophan-dependent pathway of auxin biosynthesis [26], mutants of YUCCA present aberrant flower phenotypes, short stamen filaments, and thus sterility [27]. Our results show that the expression levels of YUCCA were down-regulated significantly (Table 3 ), the flower phenotype also appears as short stamen filaments (Fig. 1b). Thus, inferring that the low expression of YUCCA is a key factor affecting the biosynthesis of auxin leading to flower sterility. In addition, we also found that the expression of SPL homolog genes as an inhibiting factor of $Y U C C A$ was up-regulated in sterile flowers (Table 4). Previous studies showed that SPL represses YUCCA gene expression to regulate the development of lateral organs [28]. Our results suggest that SPL regulates the homeostasis of auxin by inhibiting YUCCA, resulting in flower sterility.
ARF play pivotal roles in the growth of inflorescences, stamens, anthers, and pistils. Mutations in ARF6 and $A R F 8$ caused male and female infertility [29]. However, we found that the expression level of ten ARF homolog genes was up-regulated in sterile floral buds (Table 3 ). This is possibly due to the high expression of $A R F$ inhibiting auxin signaling targets [30]. Moreover, ARF acts as a positive or negative regulator by binding to the auxin response element TGTCTC [31], and it is possible that the Aux/IAA inhibitor is able to inhibit transcription through interaction with ARF [32].

Interestingly, we found that the A-class of $\mathrm{ABC}$ floral organ-identity genes $A P 1$ and $A P 2$ were up-regulated in sterile floral buds (Table 4). It has been reported that the initial expression of $A P 1$ and $A P 2$ was restricted to the first and second whorls, and was inhibited in the third and fourth whorls of flower development [33]. Thus inferring that high expression of AP1 and AP2 represses flower development in first and second whorls. Detailed mechanisms will require further study.

As an important regulator of flower development, auxin is transported to each tissue through carriers and the AUX1/LAX [34]. We found that the expression levels of 
$A U X 1 / L A X$ (Table 3) and twelve energy transfer-related genes (Table 5) were down-regulated, suggesting that the transport of auxin may be impeded. This may also be an important factor in the sterile floral buds of the tea plant.

In summary, the present work provides four key factors for the development of sterile floral buds in the tea plant. We thus present a theoretical basis for further study of mechanisms by which sterile floral buds are produced.

\section{Abbreviations}

DEGs: Differentially expressed genes; FBH: Foxiang2; GO: Gene Ontology; KEGG: Kyoto Encyclopedia of Genes and Genomes; MBH: Fudingbaicha; ZDH: Hybrid sterile flowers

\section{Acknowledgements}

This work was supported by the National Natural Science Foundation of China (31460216), Personnel Training Program of Yunnan Province (2015HB105) and State Key Laboratory of Tea Plant Biology and Utilization (SKLTOF20150105, SKLTOF20170115).

\section{Funding}

This work was funded by NSFC31460216, 2015HB105, SKLTOF20150105, and SKLTOF20170115.

\section{Availability of data and materials}

We have provided detailed information about the materials and methods in our manuscript.

\section{Authors' contributions}

$L C, M L$ and $C J$ conceived and designed the experiments. YL, HB and YS performed the experiments. LC, LX and $\mathrm{HQ}$ analyzed the data. $\mathrm{HQ}$ and $\mathrm{LC}$ wrote the paper.

\section{Ethics approval and consent to participate}

Not applicable.

\section{Consent for publication}

Not applicable.

\section{Competing interests}

The authors declare that they have no competing interests

\section{Publisher's Note}

Springer Nature remains neutral with regard to jurisdictional claims in published maps and institutional affiliations.

\section{Author details}

${ }^{1}$ Tea Research Institute, Yunnan Academy of Agricultural Sciences, Menghai 666201, China. ${ }^{2}$ Yunnan Provincial Key Laboratory of Tea Science, Menghai 666201, China. ${ }^{3}$ State Key Laboratory of Tea Plant Biology and Utilization, Anhui Agricultural University, Hefei 230036, China.

Received: 28 December 2018 Accepted: 11 April 2019

Published online: 18 April 2019

\section{References}

1. Cheng Y, Dai X, Zhao Y. Auxin biosynthesis by the YUCCA flavin monooxygenases controls the formation of floral organs and vascular tissues in Arabidopsis. Genes Dev. 2006;20(13):1790-9.

2. Tang RS, Zheng JC, Jin ZQ, et al. Possible correlation between high temperature-induced floret sterility and endogenous levels of IAA, GAs and ABA in rice (Oryza sativa L.). Plant Growth Regul. 2008:54(1):37-43,

3. Wan C, Li S, Wen L, et al. Damage of oxidative stress on mitochondria during microspores development in Honglian CMS line of rice. Plant cell Rep. 2007:26(3):373-82.

4. Smith AR, Zhao D. Sterility Caused by Floral Organ Degeneration and Abiotic Stresses in Arabidopsis and Cereal Grains. Front Plant Sci. 2016;7: 1503
5. Cheng Y, Qin G, Dai X, et al. NPY genes and AGC kinases define two key steps in auxin-mediated organogenesis in Arabidopsis. Proc Natl Acad Sci. 2008;105(52):21017-21,022

6. Okada K, Ueda J, Komaki MK, et al. Requirement of the auxin polar transport system in early stages of Arabidopsis floral bud formation. Plant Cell. 1991;3(7):677-84.

7. Ma H. Molecular genetic analyses of microsporogenesis and microgametogenesis in flowering plants. Annu Rev Plant Biol. 2005:56:393-434

8. Ito T, Wellmer F, Yu H, et al. The homeotic protein AGAMOUS controls microsporogenesis by regulation of SPOROCYTELESS. Nat. 2004;430(6997):356-60

9. Liljegren SJ, Ditta GS, Eshed Y, et al. SHATTERPROOF MADS-box genes control seed dispersal in Arabidopsis. Nat. 2000;404(6779):766-70.

10. Mayer KFX, Schoof $\mathrm{H}$, Haecker $\mathrm{A}$, et al. Role of WUSCHEL in regulating stem cell fate in the Arabidopsis shoot meristem. Cell. 1998;95(6):805-15.

11. Sorensen AM, Kröber S, Unte US, et al. The Arabidopsis ABORTED MICROSPORES (AMS) gene encodes a MYC class transcription factor. Plant J. 2003:33(2):413-23.

12. Yang $S L$, Jiang L, San Puah C, et al. Overexpression of TAPETUM DETERMINANT1 alters the cell fates in the Arabidopsis carpel and tapetum via genetic interaction with excess microsporocytes $1 /$ extra sporogenous cells. Plant Physiol. 2005;139(1):186-91.

13. Zhang W, Sun Y, Timofejeva L, et al. Regulation of Arabidopsis tapetum development and function by DYSFUNCTIONAL TAPETUM1 (DYT1) encoding a putative bHLH transcription factor. Dev. 2006;133(16):3085-95.

14. Cecchetti V, Altamura MM, Falasca G, et al. Auxin regulates Arabidopsis anther dehiscence, pollen maturation, and filament elongation. Plant Cell. 2008:20(7):1760-74.

15. Mandaokar A, Thines B, Shin B, et al. Transcriptional regulators of stamen development in Arabidopsis identified by transcriptional profiling. Plant J. 2006:46(6):984-1008

16. Szekeres $M$, Németh $K$, Koncz-Kálmán Z, et al. Brassinosteroids rescue the deficiency of CYP90, a cytochrome P450, controlling cell elongation and deetiolation in Arabidopsis. Cell. 1996;85(2):171-82.

17. Yamaguchi $N$, Wu MF, Winter CM, et al. LEAFY and polar auxin transport coordinately regulate Arabidopsis flower development. Plants. 2014:3(2):251-65.

18. Cecchetti V, Altamura M M, Falasca G, et al. Auxin regulates Arabidopsis anther dehiscence, pollen maturation, and filament elongation[J]. The Plant Cell, 2008:20(7):1760-74.

19. Grossniklaus U, Schneitz K. The molecular and genetic basis of ovule and megagametophyte development. Semin Cell Dev Biol. 1998;9(2):227-38.

20. Ainsworth C, Crossley S, Buchanan-Wollaston V, et al. Male and female flowers of the dioecious plant sorrel show different patterns of MADS box gene expression. Plant Cell. 1995;7(10):1583-98.

21. Baker LA, Habershon S. Photosynthetic pigment-protein complexes as highly connected networks: implications for robust energy transport. Proc Math Phys Eng Sci. 2017:473(2201):20170112.

22. Wei $C$, Yang $H$, Wang $\mathrm{S}$, et al. Draft genome sequence of Camellia sinensis var. sinensis provides insights into the evolution of the tea genome and tea quality. Proc Nat Acad Sci. 2018;115(18):E4151-8.

23. Li H, Huang W, Wang GL, et al. Transcriptomic analysis of the biosynthesis, recycling, and distribution of ascorbic acid during leaf development in tea plant (Camellia sinensis (L.) O. Kuntze). Sci Rep. 2017:7:46212.

24. Liu SC, Jin JQ, Ma JQ, et al. Transcriptomic analysis of tea plant responding to drought stress and recovery. PloS One. 2016;11(1):e0147306.

25. Tai Y, Wei C, Yang H, et al. Transcriptomic and phytochemical analysis of the biosynthesis of characteristic constituents in tea (Camellia sinensis) compared with oil tea (Camellia oleifera). BMC Plant Biol. 2015;15(1):190.

26. Abu-Zaitoon YM. Phylogenetic analysis of putative genes involved in the tryptophan-dependent pathway of auxin biosynthesis in rice. Appl Biochem Biotechnol. 2014;172(5):2480-95.

27. Salinas-Grenet $\mathrm{H}$, Herrera-Vásquez A, Parra $\mathrm{S}$, et al. Modulation of Auxin Levels in Pollen Grains Affects Stamen Development and Anther Dehiscence in Arabidopsis. Int J Mol Sci. 2018:19(9):2480.

28. Li LC, Qin GJ, Tsuge T, et al. SPOROCYTELESS modulates YUCCA expression to regulate the development of lateral organs in Arabidopsis. New Phytol. 2008;179(3):751-64.

29. Nagpal P, Ellis CM, Weber $H$, et al. Auxin response factors ARF6 and ARF8 promote jasmonic acid production and flower maturation. Dev. 2005; 132(18):4107-18

30. Hofmann NR. A Genome-wide Approach to Understanding a NonCanonical ARF. Plant Cell. 2017;29(8):1798-9. 
31. Gray WM, Kepinski S, Rouse D, et al. Auxin regulates SCF TIR1-dependent degradation of AUX/IAA proteins. Nat. 2001;414(6861):271.

32. Guilfoyle TJ. The PB1 domain in auxin response factor and Aux/IAA proteins: a versatile protein interaction module in the auxin response. Plant Cell. 2015;27(1):33-43.

33. Irish VF. The flowering of Arabidopsis flower development[J]. Plant J. 2010; 61(6):1014-28.

34. Novoselova ES, Mironova W, Omelyanchuk NA, et al. Mathematical modeling of auxin transport in protoxylem and protophloem of Arabidopsis thaliana root tips[]]. J Bioinform Comput Biol. 2013;11(01):1340010.

\section{Ready to submit your research? Choose BMC and benefit from:}

- fast, convenient online submission

- thorough peer review by experienced researchers in your field

- rapid publication on acceptance

- support for research data, including large and complex data types

- gold Open Access which fosters wider collaboration and increased citations

- maximum visibility for your research: over $100 \mathrm{M}$ website views per year

At $\mathrm{BMC}$, research is always in progress.

Learn more biomedcentral.com/submissions 